Sección Médica / Medical

Artículo de investigación / Research paper

\title{
Evaluación de la eficacia de novaluron 0,2 \% GR para el control de Aedes (Stegomyia) aegypti (Diptera)
}

\author{
Evaluation of the efficacy of novaluron $0.2 \%$ GR for the control of Aedes (Stegomyia) aegypti (Diptera) \\ MARCELA QUIMBAYO F. ${ }^{1}$, JULIANA PÉREZ-PÉREZ ${ }^{2}$, PAOLA A. RODRÍGUEZ-GAVIRIA ${ }^{3}$, \\ JUAN DAVID AMAYA ${ }^{4}$ GUILLERMO L. RÚA-URIBE 5
}

\begin{abstract}
${ }^{1}$ Bióloga, M. Sc., Grupo Entomología Médica, Facultad de Medicina, Universidad de Antioquia. Carrera 51D 62-29, Laboratorio 321, Medellín, Colombia, marceladelpilar45@gmail.com, https://orcid.org/0000-0001-6581-4054. ${ }^{2}$ Ingeniera Biológica, M. Sc. Grupo Entomología Médica, Facultad de Medicina, Universidad de Antioquia. Carrera 51D 62-29, Laboratorio 321, Medellín, Colombia, jperezp1017@gmail.com, https://orcid.org/0000-0002-5819-1917. ${ }^{3}$ Ingeniera Agrónoma, M. Sc. Adama Andina B.V. Sucursal Colombia. Carrera 11 No. 87 - 51 piso 4, Bogotá, D. C., Colombia. Tel: $57-16446730$ Ext. 1541 , prodriguezenator@gmail.com, https://orcid.org/0000-0003-3218-8098. ${ }^{4}$ Ingeniero Agrónomo, M. Sc. Adama Andina B.V. Sucursal Colombia. Carrera 11 No. 87 - 51 piso 4, Bogotá, D. C., Colombia. Tel: 57-1 6446730 Ext. 1541, Tel: 57-1 379 9772, juandavid.amaya@adama.com, https://orcid.org/0000-00034922-6943. ${ }^{5}$ Biólogo, Ph. D. Grupo Entomología Médica, Facultad de Medicina, Universidad de Antioquia. Carrera 51D 62-29, Laboratorio 321, Medellín, Colombia, guillermo.rua@udea.edu.co, https://orcid.org/0000-0002-9802-0194.
\end{abstract}

\begin{abstract}
Autor para correspondencia: Marcela Quimbayo. Grupo Entomología Médica, Facultad de Medicina, Universidad de Antioquia. Carrera 51D 62-29, Laboratorio 321, Medellín, Colombia, marceladelpilar45@ gmail.com, https://orcid.org/0000-0001-65814054.
\end{abstract}

Citación sugerida / Suggested citation:

QUIMBAYO F., M.; PÉREZ-PÉREZ, J.; RODRÍGUEZ-GAVIRIA, P. A.; AMAYA, J. D. RÚA-URIBE, G. L. 2019. Evaluación de la eficacia de novaluron $0,2 \%$ GR para el control de Aedes (Stegomyia) aegypti (Diptera). Revista Colombiana de Entomología 45 (1): e7812 https://doi.org/10.25100/socolen.v45i1.7812

Recibido: 4-dic-2017

Aceptado: 23-ene-2019

Publicado: 20-sep-2019

Revista Colombiana de Entomología ISSN (Impreso): 0120-0488 ISSN (En línea): 2665-4385

http://revistacolombianaentomologia.univalle.edu.co/

Open access

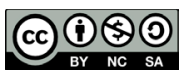

BY-NC-SA 4.0.

Publicadores / Publishers:

Sociedad Colombiana de Entomología SOCOLEN (Bogotá, D. C., Colombia) $\mathrm{http}: / /$ www.socolen.org.co Universidad del Valle (Cali, Colombia) http://www.univalle.edu.co/

(C) 2019 Sociedad Colombiana de Entomología - SOCOLEN y Universidad del Valle - Univalle
Resumen: Aedes (Stegomyia) aegypti es el vector principal de dengue, zika y chikungunya en las Américas, enfermedades de gran impacto en salud pública. De acuerdo con la Organización Mundial de la Salud (OMS), el control de estas enfermedades requiere un enfoque integral, y el control larvario hace parte de tal estrategia. Con base en ello, los Insecticidas Reguladores de Crecimiento (IRC) surgen como una alternativa eficiente para el control de los estados inmaduros de este mosquito. Con el propósito de evaluar la eficacia del IRC novaluron 0,2 $\%$ GR se determinaron en laboratorio las concentraciones letales (CL) 50, 90, 95 y 99 sobre larvas de cuarto estadio de Ae. aegypti, y en condiciones de campo se estimó el porcentaje de inhibición de emergencia empleando las CL obtenidas en laboratorio, mediante dos esquemas de manejo de agua, con recambio y sin recambio, en recipientes de $40 \mathrm{~L}$, en 30 viviendas en un barrio de Medellín (Antioquia, Colombia) con alta incidencia de dengue. Los bioensayos indicaron que las CL 50,90, 95 y 99 correspondieron a 0,$019 ; 0,055 ; 0,065$ y $0,084 \mathrm{mg} / \mathrm{L}$, respectivamente. Los resultados de campo revelan que novaluron $0,2 \% \mathrm{GR}$ inhibió eficientemente la emergencia de adultos de Ae. aegypti indicando el potencial del producto como regulador de poblaciones a muy bajas concentraciones. Se considera que el producto es de gran utilidad en los programas de prevención y control de dengue, zika y chikungunya.

Palabras clave: Culicidae, vectores de enfermedades, vigilancia, virus.

Abstract: Aedes (Stegomyia) aegypti is the main vector of dengue, zika and chikungunya in the Americas. These diseases have a significant impact on public health. According to the World Health Organization (WHO), controlling these diseases requires a comprehensive approach, and the control of larvae is a part of that strategy. Insect growth regulator (IGR) insecticides stand out as an efficient alternative for facilitating the control of Ae. aegypti at immature stages. The main goal was to evaluate the effectiveness of IGR novaluron 0.2 $\%$ GR, in the 50, 90, 95 and 99 lethal concentrations (LC) for fourth-instar larvae of $A e$. aegypti in the laboratory. In field conditions, the percentage of inhibition of emergence was estimated by using the LC levels obtained in the laboratory through two methods of water management with refill and without refill in $40 \mathrm{~L}$ recipients. The study was carried out in 30 homes in a neighborhood with a high incidence of dengue in Medellin (Antioquia, Colombia). The bioassays completed indicated that LC 50,90, 95 and 99 corresponded to $0.019,0.055,0.065$ and $0.084 \mathrm{mg} / \mathrm{L}$, respectively. The field results indicated that novaluron $0.2 \%$ GR efficiently inhibited the emergence of adult Ae. aegypti, suggesting that the product has potential as a population regulator at very low concentrations. The product is considered extremely useful for programs to prevent and control dengue, zika and chikungunya.

Keywords: Culicidae, disease vectors, surveillance, virus.

\section{Introducción}

En las últimas décadas, las enfermedades de transmisión vectorial han cobrado mayor importancia en salud pública en las diferentes regiones tropicales y subtropicales del mundo. En particular, se ha evidenciado una rápida expansión geográfica de los virus dengue, zika y chikungunya, y de los vectores que las transmiten. De acuerdo con la 
Organización Panamericana de la Salud (OPS), para dengue se ha registrado un incremento de 30 veces en la incidencia global en los últimos 50 años (PAHO 2008), mientras que zika y chikungunya presentan un fuerte potencial epidémico en cada país en donde se registran casos de estas enfermedades (Rincón Silva y Rincón Silva 2016).

En Colombia, se ha observado que el número de casos de dengue en la última década se ha incrementado paulatina pero significativamente, incluso, las epidemias se han vuelto más frecuentes; esta situación epidemiológica también ha sido evidente en otros países de la región (Padilla et al. 2012).

De acuerdo con el Instituto Nacional de Salud de Colombia (INS), el promedio anual del número de casos notificados de dengue en la última década es de, aproximadamente, cien mil de los cuales, menos del $1 \%$ corresponden a la forma grave de la enfermedad. De estos casos, departamentos como Valle, Antioquia, Santander y Tolima, son los que aportan el mayor porcentaje de notificaciones (INS 2014; INS 2016).

En cuanto a chikungunya y zika se registran casos en el país desde el 2014 y 2015, respectivamente (SIVIGILA 2014; MinSalud 2015), impactando fuertemente ciudades de la costa caribe, en donde el número de personas afectadas superó el $80 \%$ de la población local (INS 2014; INS 2016). Particularmente, para zika se ha indicado que sus implicaciones en salud son más graves que las de chikungunya, debido a que puede ser causante de microcefalia en neonatos de madres infectadas con el virus. También se ha observado asociación de zika con Guillain-Barré, una enfermedad del sistema nervioso, en la cual el sistema inmune del paciente afecta sus propias neuronas, causando debilidad muscular y en ocasiones parálisis (Cao-Lormeau et al. 2016).

A pesar de la relevancia de estas enfermedades en el contexto de salud mundial, hasta el momento no existe una vacuna. Aunque para dengue se han realizado algunos estudios para el desarrollo de la vacuna aún no ha sido autorizada por la OMS para su uso en pacientes (López-Gatell et al. 2016). Es por esto que para mitigar el impacto de estas enfermedades se requiere un enfoque integral, que cuente no solo con el uso intensivo de adulticidas sino también la implementación de larvicidas y la participación comunitaria, ya que el control vectorial ha sido la estrategia más frecuentemente empleada (San Martin 2009).

Estas enfermedades son causadas por virus transmitidos principalmente por Aedes (Stegomyia) aegypti (Linneaus, 1762) (Diptera: Culicidae), mosquito que se distribuye, por debajo de los $2.200 \mathrm{msnm}$, en los más importantes centros urbanos del país (San Martin 2009), aunque reportes recientes lo ubican en alturas cercanas a los $2.350 \mathrm{msnm}$ (Ruiz-López et al. 2016). Este vector es de hábitos domiciliarios y se cría principalmente en recipientes con agua detenida relativamente limpias, que la población humana emplea para diferentes usos domésticos (Kouri 2006).

Con base en lo anterior, el control vectorial se ha enfocado en la eliminación de sitios de cría, el uso de adulticidas intra y peridomiciliario y al empleo de larvicidas. Sin embargo, en Colombia y en otros países de la región, se ha observado que algunas poblaciones del vector son resistentes a los insecticidas frecuentemente empleados en los programas de salud (Conde et al. 2015; Santacoloma et al. 2012; Rawlins y Wan 1995; Maestres et al. 2009; Álvarez et al. 2006). Particularmente para el país, se registró que Ae. aegypti es resistente al organofosforado temefos (Suárez et al. 1996; Santacoloma et al. 2012; Conde et al. 2015); desde entonces se ha reportado resistencia a este larvicida en los departamentos de Antioquia, Cundinamarca, Santander, Caquetá, Meta, Guaviare, Sucre, Huila, Cauca y Nariño (Anaya et al. 2007; Fonseca et al. 2007; Salazar et al. 2007; Santacoloma et al. 2007; Conde et al. 2015). También se ha registrado resistencia a insecticidas como DDT, propoxur y lamdacialotrina en cuatro departamentos del sur oeste del país (Ocampo et al. 2011).

Debido a la resistencia a insecticidas desarrollada por $A e$. aegypti y considerando que para mitigar el impacto de estas enfermedades se requiere un enfoque integral, con alternativas más amigables con el ambiente, pero que permitan una eficiente reducción vectorial, han surgido diferentes estrategias para el control de mosquitos. Una de estas iniciativas son los Insecticidas Reguladores de Crecimiento (IRC), y entre estos, novaluron $0,2 \% \mathrm{GR}$, el cual es un producto que inhibe la síntesis de quitina, afectando el ciclo de vida de los insectos.

Los IRC basados en novaluron actúan por ingestión y contacto, causando una anormal deposición endocuticular, lo que conlleva a fallas en el proceso de muda (Hilton y VanBuskirk 2002). Estos IRC han sido evaluados con resultados muy satisfactorios en poblaciones de Ae. aegypti (Mulla y Darwazeh 1988; Nwankwo et al. 2011) y varias especies de Anopheles (Arredondo-Jiménez y Valdez-Delgado 2006; Chanda et al. 2013), pero en formulación de concentrado emulsionable (EC, por sus siglas en inglés). El diseño de una nueva formulación del producto (granulado) requiere la determinación de su eficacia en condiciones de laboratorio y campo. Para ello y siguiendo la guía de la WHO (2005a), se realizó la presente evaluación con el propósito de determinar en condiciones de laboratorio y campo, la eficacia de novaluron $0,2 \%$ GR sobre larvas de Ae. aegypti.

\section{Materiales y métodos}

Evaluación de novaluron 0,2 \% GR en condiciones controladas de laboratorio. Las larvas empleadas en el estudio se obtuvieron de una colonia de mosquitos establecida en el insectario del Laboratorio de Entomología Médica de la Facultad de Medicina, Universidad de Antioquia, la cual se originó a partir de huevos recolectados mediante ovitrampas ubicadas en diferentes barrios de Medellín. Los ejemplares fueron mantenidos a una temperatura de $25+/-2{ }^{\circ} \mathrm{C}$, una humedad relativa del $70+/-10 \%$, un fotoperíodo 12:12 luz:oscuridad, y alimentados siguiendo el protocolo del American Mosquito Control Association (1970).

Determinación de CL 50, CL 90, CL 95 y CL 99. Para las evaluaciones se emplearon larvas F2 de Ae. aegypti de estadios 1 y 4 (L1 y L4). Debido a que la formulación granulada de novaluron $0,2 \%$ GR no había sido evaluada con anterioridad en Colombia, se consideraron los resultados de evaluaciones previas realizadas en Brasil para establecer la concentración de referencia (Fontoura et al. 2012). A partir de ella se evaluaron 14 concentraciones que fluctuaron entre $50 \mathrm{mg} / \mathrm{L}$ y $0,00625 \mathrm{mg} / \mathrm{L}$. De cada concentración se evaluaron cuatro réplicas, cada una de las cuales contenía 25 larvas, con su respectivo control, el cual consistió en larvas mantenidas bajo las mismas condiciones del tratamiento, pero sin adición del producto. Cada ensayo se repitió cuatro veces. Las evaluaciones se realizaron siguiendo los métodos propuestos por la WHO (2005a). Cada 24 horas se registró el número de larvas, 
pupas y adultos vivos y muertos, hasta una semana después de haber emergido el $100 \%$ de los mosquitos en el control. Los especímenes muertos fueron retirados luego de contarlos. Con los datos de mortalidad se calcularon las concentraciones letales CL 50, 90, 95 y 99 mediante análisis Probit. A partir de los resultados obtenidos con larvas L4, se seleccionaron las tres concentraciones más bajas para evaluar la eficacia del producto en larvas L1.

Evaluación de novaluron 0,2 \% GR en condiciones de campo. Con base en los reportes de casos de dengue de la ciudad de Medellín, suministrados por la Secretaría de Salud Municipal (SIVIGILA 2014), se seleccionó el barrio Campo Valdés 1, en la comuna 4 en el sector nororiental de la ciudad, para realizar la evaluación del producto bajo condiciones de campo. Este barrio cuenta con aproximadamente 4.500 viviendas, la mayoría de estrato socioeconómico tres (Convenio DANE - Municipio de Medellín 2009).

Evaluación de concentraciones letales. Se seleccionaron aleatoriamente 30 viviendas, en cada una de ellas se ubicaron tres canecas de $40 \mathrm{~L}$, dos de ellas fueron tratamientos (con producto) y una el control. Los tratamientos se basaron en dos esquemas de manejo de agua, 1. "sin" recambio de agua y 2. con recambio de agua. Para el primer esquema solo el agua que se evaporó fue reemplazada semanalmente, mientras que para el segundo, una vez por semana se reemplazó el $50 \%$ de la cantidad de agua, lo cual conllevó a que en dos semana se reemplazara el $100 \%$ del contenido inicial. Con el esquema de reemplazo de agua se pretendió simular el empleo diario que puede hacerse en una vivienda. Las tres canecas ubicadas por vivienda (tratamiento "sin" recambio de agua, tratamiento con recambio de agua y control) fueron mantenidas al $90 \%$ de la capacidad total de almacenamiento.

Las concentraciones de novaluron $0,2 \%$ GR evaluadas en condiciones de campo fueron las CL 90, 95 y 99 obtenidas a partir de los bioensayos de laboratorio con L4. En cada grupo de 10 viviendas se evaluó una de las CL. El producto fue aplicado el primer día de los experimentos. Para garantizar la presencia del vector, cada quince días se vertieron en cada caneca un lote de 100 larvas de primer y/o segundo estadio. Una vez por semana se registró el número de pupas por cada caneca y se colectaron los mosquitos adultos emergidos. Las pupas obtenidas fueron transportadas hasta el insectario del Grupo Entomología Médica, de la facultad de Medicina de la Universidad de Antioquia, (GEM), en donde bajo condiciones controladas de laboratorio $\left(\mathrm{T}^{\circ} 25+/-2{ }^{\circ} \mathrm{C}\right.$, HR $\left.70+/-10 \%\right)$, se mantuvieron hasta la emergencia del mosquito adulto. El muestreo de adultos y pupas se realizó durante 12 semanas, o hasta que la mortalidad observada en los tratamientos fue similar a la del control.

Tamaño alar de los mosquitos emergidos. Considerando que algunos insecticidas, al reducir la densidad larval de los sitios de cría, podrían seleccionar mosquitos de mayor talla y por tanto con mayor capacidad de dispersión, se propuso comparar el tamaño de los mosquitos emergidos en las diferentes CL y en el control. Para ello, de acuerdo con Nasci (1986, 1987); Nasci y Mitchell (1994) y Sumanochitrapon et al. (1998), se consideró que el tamaño alar es un buen indicador de la talla del mosquito. Para el cálculo de la morfometría alar se tomó una muestra de los mosquitos hembra (entre 24 y 30) que emergieron de las diferentes CL y el control, a

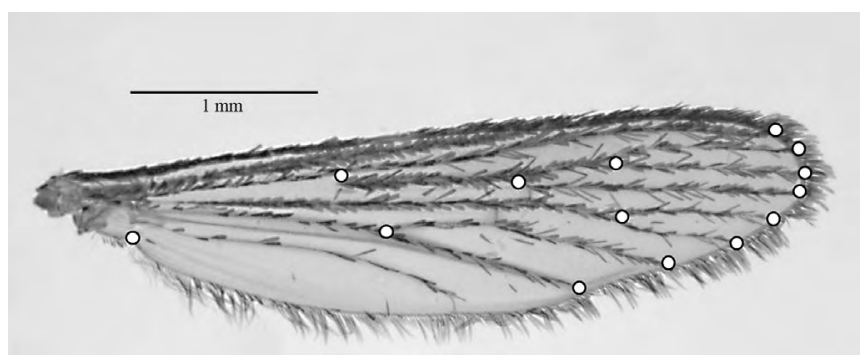

Figura 1. Ala derecha de Ae aegypti indicando los 14 puntos anatómicos empleados para calcular el tamaño centroide.

las cuales se les retiró el ala derecha. Posteriormente se digitalizaron 14 puntos anatómicos tipo I (Bookstein 1991), los cuales representaron intersecciones vena-vena y finalización de vena en el borde del ala (Fig. 1). Estos puntos se seleccionaron de acuerdo con lo propuesto por Jaramillo-O. et al. (2015). Con los puntos anatómicos se calculó el tamaño centroide de los tratamientos y el control, posteriormente fueron comparados entre sí.

Análisis de la información. Para las diferentes concentraciones evaluadas en condiciones de laboratorio se estimó el porcentaje de mortalidad, dado por la relación entre el número de larvas muertas sobre las expuestas, por cien. También se calculó el porcentaje de inhibición de la emergencia (IE) mediante la siguiente fórmula:

$$
\% \mathrm{IE}=100-\left(100 \times\left(\frac{\% \text { Emergencia Tto. }}{\% \text { Emergencia Control }}\right)\right)
$$

Se empleó la fórmula Abbott para corregir los datos de mortalidad en el control cuando fue necesario y se empleó el análisis por el modelo Probit (Finney 1971) para determinar las diferentes CL que se evaluaron en campo. Allí, semanalmente se registró el número de mosquitos emergidos en tratamientos y control, y se determinó normalidad de los datos. Debido a que no cumplieron los supuestos de normalidad, se compararon medias geométricas e intervalos de confianza del $95 \%$. En cuanto al análisis morfométrico, se utilizó la prueba no paramétrica de Kruskall-Wallis y para las comparaciones pareadas se empleó la prueba Mann-Whitney. Todos los análisis estadísticos fueron realizados empleando el software Statgraphics Centurion XV v. 15.2.06.

Consideraciones éticas. Las personas que accedieron a participar en la evaluación de campo firmaron un consentimiento informado que garantizaba que no habría ninguna consecuencia en salud por la tenencia de las canecas, debido a que no se permitiría la emergencia de los mosquitos en el lugar de residencia.

\section{Resultados y discusión}

Evaluación de novaluron 0,2 \% GR en condiciones controladas de laboratorio. El novaluron $0,2 \%$ GR afectó significativamente la emergencia de adultos de Ae. aegypti. Este producto impidió que los mosquitos adultos lograran desprenderse de la exuvia, lo cual conllevó a que los ejemplares no completaran la metamorfosis. Similar efecto del producto 
Tabla 1. Resultados de la evaluación de novaluron $0,2 \%$ GR a diferentes concentraciones en larvas L4 de Aedes aegypti bajo condiciones controladas de laboratorio.

\begin{tabular}{|c|c|c|c|c|c|c|c|c|}
\hline \multirow{2}{*}{$\begin{array}{c}\text { Concentración } \\
(\mathrm{mg} / \mathrm{L})\end{array}$} & \multicolumn{2}{|c|}{ Tratamientos } & \multicolumn{2}{|c|}{ Control } & \multirow{2}{*}{$\begin{array}{c}\% \\
\text { Mortalidad }\end{array}$} & \multirow{2}{*}{$\begin{array}{c}\% \\
\text { Emergencia } \\
\text { Expuestos }\end{array}$} & \multirow{2}{*}{$\begin{array}{c}\% \\
\text { Inhibición } \\
\text { Emergencia }\end{array}$} & \multirow{2}{*}{$\begin{array}{c}\% \\
\text { Inhibición } \\
\text { Emergencia }\end{array}$} \\
\hline & Vivos & Muertos & Vivos & Muertos & & & & \\
\hline $\mathrm{C} 1: 50,0$ & 0 & 300 & 75 & 0 & 100 & 0 & 100 & 100 \\
\hline $\mathrm{C} 2: 25,0$ & 2 & 298 & 61 & 14 & 99,3 & 0,7 & 81,3 & 99,2 \\
\hline C3: 12,5 & 0 & 300 & 69 & 6 & 100 & 0 & 92 & 100 \\
\hline C4: 6,30 & 1 & 299 & 74 & 1 & 99,7 & 0,3 & 98,7 & 99,7 \\
\hline C5: 3,15 & 0 & 300 & 72 & 3 & 100 & 0 & 96 & 100 \\
\hline C6: 1,60 & 1 & 299 & 73 & 2 & 99,7 & 0,3 & 97,3 & 99,7 \\
\hline C7: $1,00^{*}$ & 0 & 400 & 99 & 1 & 100 & 0 & 99 & 100 \\
\hline C8: 0,40 & 28 & 372 & 100 & 0 & 93 & 7 & 100 & 93 \\
\hline C9: 0,20 & 32 & 368 & 100 & 0 & 92 & 8 & 100 & 92 \\
\hline C10: $0,10^{*}$ & 16 & 384 & 100 & 0 & 96 & 4 & 100 & 96 \\
\hline C11: $0,05^{*}$ & 19 & 381 & 100 & 0 & 95,3 & 4,8 & 100 & 95,3 \\
\hline C12: $0,025^{*}$ & 107 & 293 & 100 & 0 & 73,3 & 26,8 & 100 & 73,3 \\
\hline C13: $0,0125^{*}$ & 252 & 148 & 100 & 0 & 37 & 63 & 100 & 37 \\
\hline C14: $0,00625^{*}$ & 331 & 69 & 100 & 0 & 17,3 & 82,8 & 100 & 17,3 \\
\hline
\end{tabular}

* Concentraciones empleadas para calcular las CL de acuerdo con el modelo probit.

también se observó en los estados de larva y pupa, en las cuales la exuvia permaneció adherida limitando el nado y la ecdisis. Las concentraciones entre 50,0 y $1,0 \mathrm{mg} / \mathrm{L}$ generaron mortalidades superiores al $99 \%$. Resultados similares se observaron cuando se calculó el porcentaje de inhibición de la emergencia (Tabla 1). Debido a que los resultados de las concentraciones 0,40 y $0,20 \mathrm{mg} / \mathrm{L}$ no fueron consistentes con los obtenidos en la concentración $0,10 \mathrm{mg} / \mathrm{L}$ y las sucesivas, se consideró no incluirlas para calcular las CL 50, 90, 95 y 99, las cuales correspondieron respectivamente a 0,$019 ; 0,055$; 0,065 y $0,084 \mathrm{mg} / \mathrm{L}$ (Fig. 2). Con relación a las evaluaciones del producto en L1, se observó que los resultados fueron similares a los registrados en L4 (Tabla 2), sin embargo, y como era de esperarse, los estadíos larvales L1 fueron más susceptibles, lo cual fue evidente al observar un mayor porcentaje de mortalidad e inhibición de la emergencia en las tres concentraciones evaluadas. Estos resultados evidencian que las concentraciones evaluadas de novaluron $0,2 \% \mathrm{GR}$ afectaron la metamorfosis de los estadíos inmaduros de $A e$. aegypti, disminuyendo significativamente la emergencia del vector. Resultados similares han sido reportados por Arredondo-Jiménez y Valdez-Delgado (2006) quienes encontraron que este producto tuvo efecto contra larvas de Anopheles, Aedes y Culex, en Chiapas, México, concluyendo que novaluron es eficaz para el control de larvas de estas tres especies de vectores, sugiriendo que podría ser empleado como un componente más en los programas de control y prevención de dengue y malaria. Por otro lado, Mulla et al. (2003) también observaron un notable efecto larvicida, principalmente en el segundo estadío, evidenciando que las pupas también son afectadas por la actividad del IRC, dada la disminución en la emergencia de mosquitos adultos, aunque las concentraciones de Mulla y colaboradores fueron menores $(0,25$ a $1,0 \mu \mathrm{g} / \mathrm{L}$ ) que las del presente estudio, es claro que novaluron como ingrediente activo presenta una fuerte actividad larvicida y de inhibición de la emergencia. Es posible que las diferencias observadas se deban al tipo de formulación empleada en ambos estudios. En cuanto a las alteraciones morfológicas que se presentaron en las larvas para este estudio, se observó que las exuvias no se desprendieron completamente de las
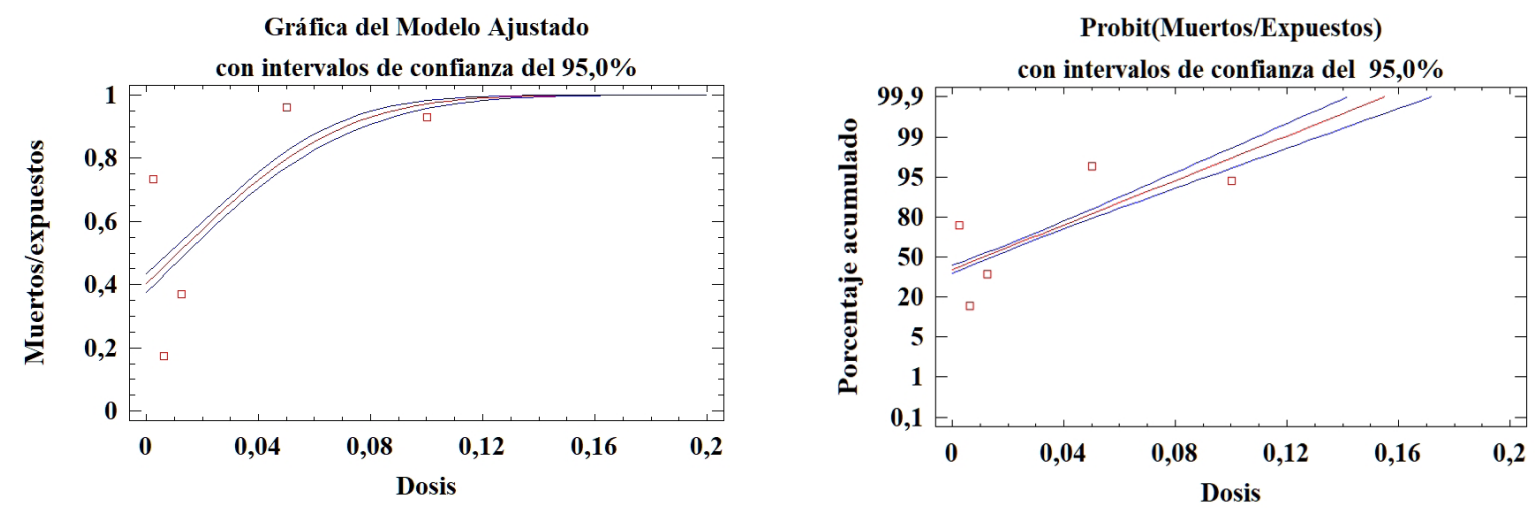

Figura 2. Modelo Probit para el cálculo de las CL de novaluron 0,2 \% GR sobre Aedes aegypti. 
Tabla 2. Resultados de la evaluación de novaluron $0,2 \%$ GR a diferentes concentraciones sobre en larvas L1 de Aedes aegypti bajo condiciones controladas de laboratorio.

\begin{tabular}{|c|c|c|c|c|c|c|c|c|}
\hline \multirow{2}{*}{$\begin{array}{c}\text { Concentración } \\
(\mathrm{mg} / \mathrm{L})\end{array}$} & \multicolumn{2}{|c|}{ Tratamientos } & \multicolumn{2}{|c|}{ Control } & \multirow{2}{*}{$\begin{array}{c}\% \\
\text { Mortalidad }\end{array}$} & \multirow{2}{*}{$\begin{array}{c}\% \\
\text { Emergidos } \\
\text { Expuestos }\end{array}$} & \multirow{2}{*}{$\begin{array}{c}\% \\
\text { Emergencia } \\
\text { Control }\end{array}$} & \multirow{2}{*}{$\begin{array}{c}\% \\
\text { Inhibición } \\
\text { Emergencia }\end{array}$} \\
\hline & Vivos & Muertos & Vivos & Muertos & & & & \\
\hline C12: 0,025 & 0 & 400 & 100 & 0 & 100 & 0 & 100 & 100 \\
\hline C13: 0,0125 & 0 & 400 & 100 & 0 & 100 & 0 & 100 & 100 \\
\hline C14: 0,00625 & 177 & 223 & 100 & 0 & 55,8 & 44,3 & 100 & 55,8 \\
\hline
\end{tabular}

larvas o pupas, además cuando los adultos lograban emerger no conseguían emprender el vuelo, resultados similares a los encontrados por Farnesi et al. (2012) en donde el estudio de los efectos de novaluron sobre la síntesis de quitina determinó que este IRC afectó significativamente el contenido de quitina, induciendo en la larva una cutícula discontinua y alterada en algunas regiones; demostrando además, que novaluron induce a la mortalidad inmadura de Ae. aegypti, y altera las proporciones hembra-macho en los adultos. El empleo de los IRC se ha consolidado rápidamente en el control vectorial, debido a su baja toxicidad, alto efecto residual y elevada eficacia en diferentes poblaciones de mosquitos (Mulla et al. 2003; Nwankwo et al. 2011; Arredondo-Jiménez y ValdezDelgado 2006). Los resultados de este estudio muestran que el novaluron representa una alternativa para el control del mosquito transmisor de dengue, zika y chikungunya, dada la resistencia reportada a otros larvicidas.

\section{Evaluación de novaluron $0,2 \%$ GR en condiciones de} campo. Las CL evaluadas en condiciones de campo correspondieron a 0,$055 ; 0,065$ y $0,084 \mathrm{mg} / \mathrm{L}$ (CL 90,95 y 99, respectivamente). Sin embargo, la calidad del agua de los dos esquemas difirió notablemente entre ellos, lo cual no permitió observar el efecto comparativo de novaluron $0,2 \% \mathrm{GR}$ entre el tratamiento con recambio y "sin" recambio de agua. El agua de este último esquema presentó características muy similares a las del control. Con base en lo anterior, no se incluyó el tratamiento con recambio en los análisis. Estas condiciones en la calidad del agua ya habían sido reportadas por Fulcher et al. (2016), estudio de campo realizado en Florida, EE. UU., en el que se empleó novaluron para controlar Culex quinquefasciatus Say, 1823 en criaderos urbanos, y la efica-

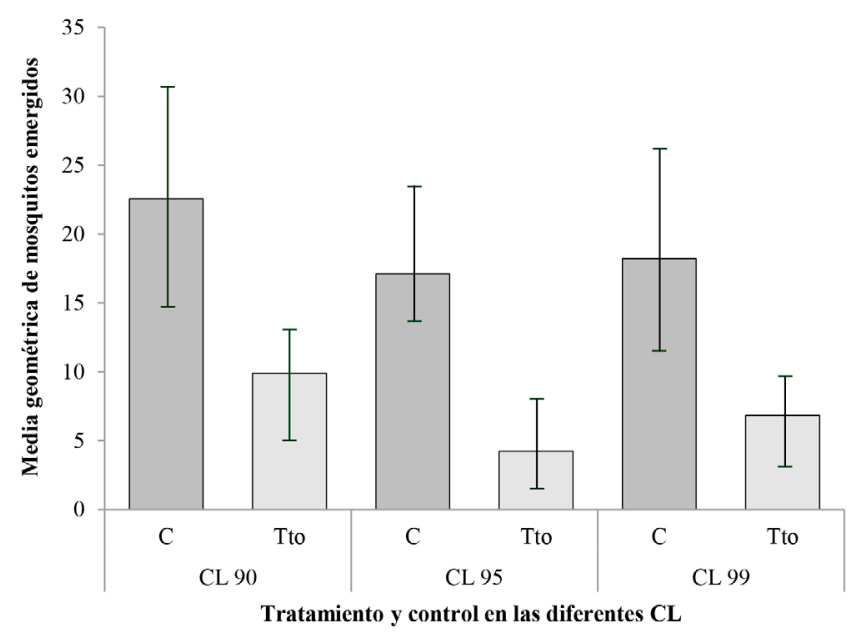

Figura 3. Efecto de CL $90(0,055 \mathrm{mg} / \mathrm{L}), 95(0,065 \mathrm{mg} / \mathrm{L})$ y $99(0,084$ $\mathrm{mg} / \mathrm{L}$ ) de novaluron $0,2 \%$ GR sobre Aedes aegypti en condiciones de campo. cia de este producto se vio afectada por las características del agua, como volumen y presencia de desechos orgánicos, las cuales pueden tener efectos sobre la eficiencia del IRC. Para las diferentes CL evaluadas en campo se observó que novaluron $0,2 \%$ GR inhibió eficientemente la emergencia
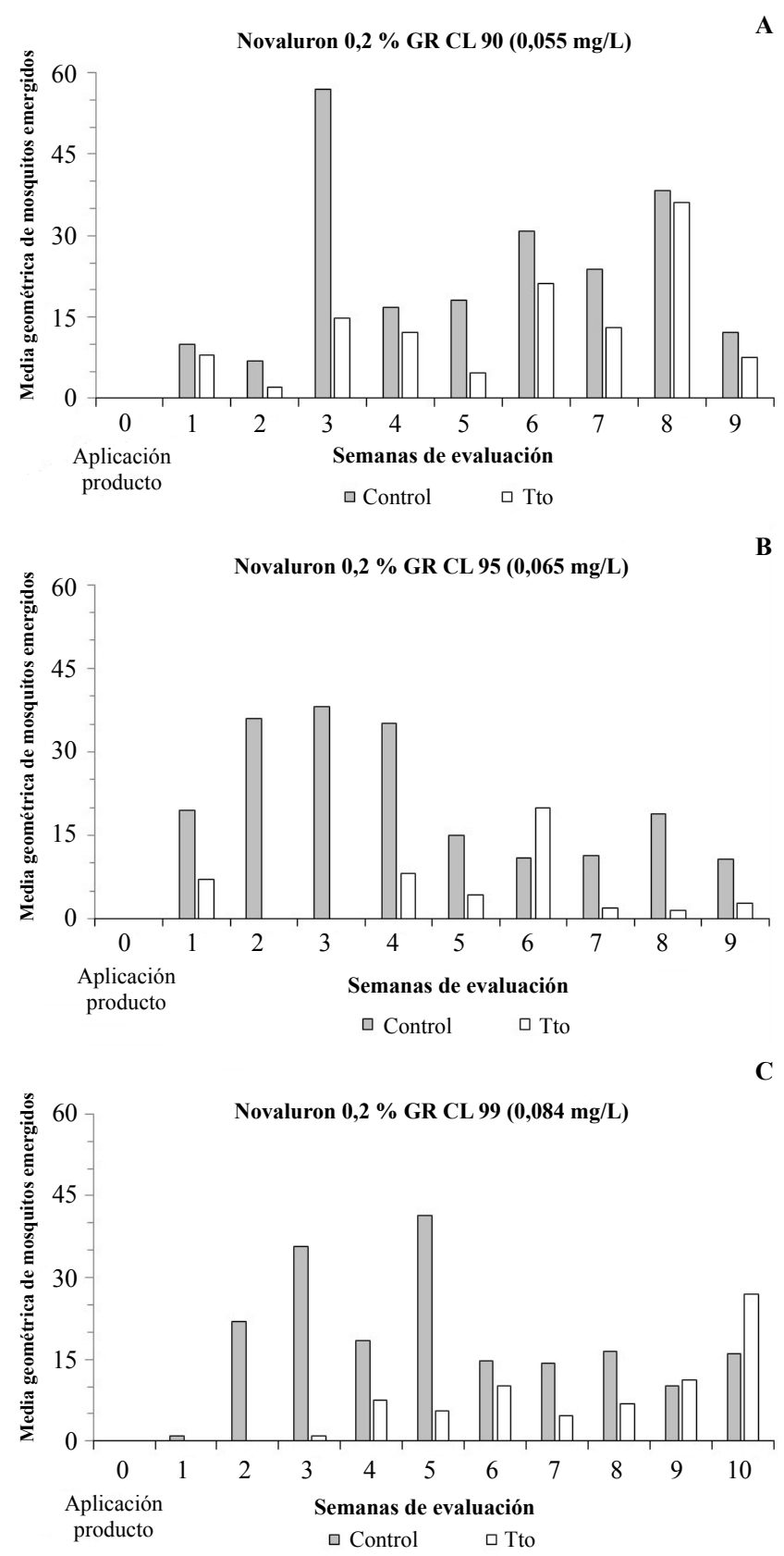

Figura 4. Variación temporal del efecto de novaluron $0,2 \% \mathrm{GR}$ a diferentes CL sobre Aedes aegypti en condiciones de campo. 
de $A$ e. aegypti (Fig. 3). De acuerdo con el análisis, la emergencia de mosquitos en los controles fue significativamente mayor que en el tratamiento sin recambio de agua, en este último, no se observó diferencia estadística en el promedio de la emergencia para las diferentes CL evaluadas. Cuando se examinó el efecto temporal del producto se observó que para las CL 90 y 95, novaluron 0,2\% GR actuó eficientemente en promedio hasta la sexta semana. Sin embargo, cuando se analizó la duración del efecto para la CL 99, se encontró que el producto presentó una mayor residualidad, llegando a ser eficiente por más de dos meses (Fig. 4). Respecto a los resultados de residualidad del producto en este estudio, se observó que podía llegar a ser eficiente hasta la décima semana de exposición en condiciones de campo, resultados comparables con los hallazgos de estudios de campo en Tailandia, por Tawatsin et al. (2007), sobre criaderos urbanos de $C$. quinquefasciatus, donde reportaron que el producto era efectivo hasta siete semanas después de su aplicación. Por otro lado, Jambulingam et al. (2009) registraron que novaluron $10 \%$ EC se puede utilizar para el control de larvas de $C$. quinquefasciatus en diferentes tipos de criaderos, a diferentes dosis e intervalos de tiempo $\left(1 \mathrm{mg} \mathrm{IA} / \mathrm{m}^{2}\right.$ cada 10 días, en pozos de aguas negras, $5 \mathrm{mg} \mathrm{IA} / \mathrm{m}^{2}$ cada 30 días en pozos abandonados y $10 \mathrm{mg} \mathrm{IA} / \mathrm{m}^{2}$ cada dos semanas en los desagües), con estos tratamientos demostraron que incluso a la concentración más baja, el IRC impidió la aparición de adultos durante al menos una semana en criaderos urbanos y durante un mes en criaderos abandonados. Además en estudios realizados en Brasil, por Fontoura et al. (2012), en ensayos simulados en campo con reemplazo parcial de agua para evaluar la persistencia de novaluron, se encontró un efecto residual de ocho semanas bajo condiciones de área interior y persistió durante cinco a seis semanas en los ensayos realizados en área externa. En cuanto al análisis del tamaño alar de los mosquitos, se observó que un aumento en la concentración del producto afectó el tamaño alar, siendo los ejemplares sometidos a mayores concentraciones los que presentaron un menor valor promedio $(3,38$ y 3,53 $\mathrm{mm}$ para CL 99 y 90, respectivamente). Sin embargo, al realizar el análisis estadístico no se determinaron diferencias significativas en el tamaño alar de los mosquitos emergidos entre las diferentes CL y el control (Fig. 5). En este estudio no se encontró una diferencia estadísticamente significativa en el tamaño de los mosquitos, la leve diferencia observada en el tamaño al incrementar la concentración podría ser explicada por la capacidad de retrasar el crecimiento de las larvas y afectar la biosíntesis y deposición de quitina, lo que podría

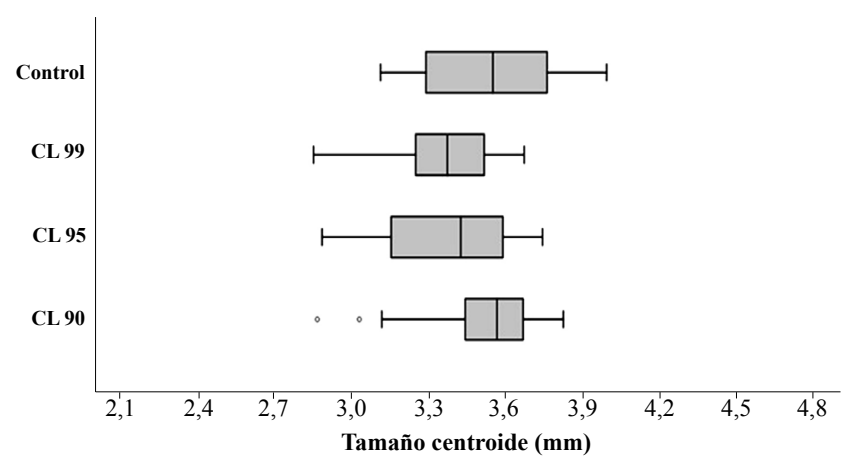

Figura 5. Comparación del tamaño centroide de alas de Aedes aegypti entre diferentes CL de novaluron $0,2 \%$ GR y control. conllevar a un tamaño inferior en los mosquitos que consiguen completar su ciclo de vida.

La principal forma de control de dengue, zika y chikungunya es la eliminación del vector, mediante la prolongada y constante utilización de insecticidas; es así como su uso indiscriminado ha generado que las poblaciones del mosquito $A e$. aegypti presente resistencia a los diferentes tipos de larvicidas y adulticidas empleados tradicionalmente en los programas de control vectorial municipales (Alvarez et al. 2006; Braga et al. 2004; Grisales et al. 2013; Conde et al. 2015), los IRC son eficaces y tienen efecto residual en las evaluaciones de campo, además se ha reportado que tienen baja toxicidad en mamíferos, aves y peces (WHO 2005b). Los IRC son una alternativa al manejo integrado de vectores de importancia en salud pública, particularmente, novaluron $0,2 \%$ GR puede ser empleado como alternativa en los programas de control vectorial de dengue en Colombia.

\section{Agradecimientos}

Los autores deseamos expresar nuestro profundo agradecimiento a la ADAMA Andina B.V Sucursal Colombia por la financiación de este estudio. A las personas que permitieron el ingreso a sus viviendas para realizar la fase de campo. También nuestros agradecimientos al Grupo Entomología Medica, GEM, de la Facultad de Medicina, Universidad de Antioquia.

\section{Literatura citada}

ÁlVAREZ, L.; BRICEÑO, A.; OVIEDO, M. 2006. Resistencia al temefos en poblaciones de Aedes aegypti (Diptera: Culicidae) del occidente de Venezuela. Revista Colombiana de Entomología 32: 172-175.

AMERICAN MOSQUITO CONTROL ASSOCIATION (AMCA). 1970. Manual for mosquito rearing and experimental techniques. Bulletin $\mathrm{N}^{\mathrm{0}} 5.112 \mathrm{p}$.

ANAYA, Y.; COCHERO, S.; REY, G.; SANTACOLOMA, L. 2007. Evaluación de la susceptibilidad a insecticidas en Aedes aegypti capturados en Sincelejo. Memorias XIII Congreso colombiano de parasitología y medicina tropical. Biomédica 27: 257.

ARREDONDO-JIMÉNEZ, J. I.; VALDEZ-DELGADO, K. M. 2006. Effect of novaluron (Rimon ${ }^{\circledR} 10 \mathrm{EC}$ ) on the mosquitoes Anopheles albimanus, Anopheles pseudopunctipennis, Aedes aegypti, Aedes albopictus and Culex quinquefasciatus from Chiapas, Mexico. Medical and Veterinary Entomology 20: 377-387. https://doi.org/10.1111/j.1365-2915.2006.00656.x

BOOKSTEIN F. L. 1991. Morphometric tools for landmark data. Geometry and biology. Cambridge University Press. https://doi. org/10.1017/CBO9780511573064

BRAGA, I. A.; LIMA, J.; SOARES, S.; VALLE, D. 2004. Aedes aegypti resistance to temephos during 2001 in several municipalities in the states of Rio de Janeiro, Sergipe, and Alagoas, Brazil. Memórias do Instituto Oswaldo Cruz 99 (2): 199-203. https:// dx.doi.org/10.1590/S0074-02762004000200015

CAO-LORMEAU, V. M.; BLAKE, A.; MONS, S.; LASTÈRE, S.; ROCHE, C.; VANHOMWEGEN, J.; DUB, T. 2016. GuillainBarré syndrome outbreak associated with Zika virus infection in French Polynesia: a case-control study. Lancet 387: 1531-1539. https://doi.org/10.1016/S0140-6736(16)00562-6

CHANDA, E.; KANDYATA, A.; CHANDA, J.; BABOO, K. S. 2013. Bio-efficacy and persistence evaluation of Dimilin ${ }^{\circledR}$ GR-2 $\%$ and Mosquiron ${ }^{\circledR} 10 \mathrm{EC}$ insect growth regulators against Anopheles gambiae s.l. (Diptera: Culicidae) larvae. Journal Biomedical Science and Engineering 6: 11-16. https://doi.org/10.4236/ jbise.2013.67A1003 
CONDE, M.; ORJUELA, L.; CASTWLLANOS, C.; HERRERAVARELA, M.; LICASTRO, S.; QUIÑONES, M. 2015. Evaluación de la sensibilidad a insecticidas en poblaciones de Aedes aegypti (Diptera: Culicidae) del departamento de Caldas, Colombia, en 2007 y 2011. Biomédica 35 (1): 43-52. doi: http:// dx.doi.org/10.7705/biomedica.v35i1.2367

CONVENIO DANE - MUNICIPIO DE MEDELLÍN. 2009. Municipio de Medellín. Proyecciones de población 2006 - 2015. Por comunas y corregimientos. Resultados Convenio Interadministrativo DANE- Municipio de Medellín, Colombia. 395 p.

FARNESI, L. C.; BRITO, J. M.; LINSS, J. G.; PELAJO-MACHADO, M.; VALLE, D. 2012 Physiological and morphological aspects of Aedes aegypti developing larvae: Effects of the chitin synthesis inhibitor novaluron. PLoS ONE 7 (1): e30363. https:// doi.org/10.1371/journal.pone.0030363

FINNEY D. J. 1971. Probit Analysis, 3ra ed, Cambridge University Press, Cambridge. 333 p.

FONSECA, I.; BOLAÑOS, D.; GÓMEZ, W.; QUIÑONES, M. 2007. Evaluación de la susceptibilidad de larvas de Aedes aegypti a insecticidas en el departamento de Antioquia. Memorias XIII Congreso colombiano de parasitología y medicina tropical. Biomédica 27 (2): 176.

FONTOURA, N. G.; BELLINATO, D. F.; VALLE, D.; LIMA, J. B. P. 2012. The efficacy of a chitin synthesis inhibitor against field populations of organophosphate-resistant Aedes aegypti in Brazil. Memorias do Instituto Oswaldo Cruz 107: 387-395. https:// doi.org/10.1590/S0074-02762012000300014

FULCHER, A.; RUI-DE XUE, J. M. S.; SMITH, M. L.; GAINES, M. K.; WEAVER, J. H. 2016. Field evaluation of mosquiron 0.12 crd against Culex quinquefasciatus in storm drains, downtown St. Augustine, Florida. Technical Bulletin of the Florida Mosquito Control Association 10: 54.

GRISALES, N.; POUPARDIN, R.; GÓMEZ, S.; FONSECA-GONZÁLEZ, I.; RANSON, H.; LENHART, A. 2013. Temephos resistance in Aedes aegypti in Colombia compromises dengue vector control. PLOS Neglected Tropical Diseases 7: (e2438) https:// doi.org/10.1371/journal.pntd.0002438. https://doi.org/10.1371/ journal.pntd.0002438

HILTON, R. J.; VANBUSKIRK, P. D. 2002. Evaluation of codling moth controls: dimilin, novaluron and avaunt. pp. 1-8. In: Proceedings of the 76th Annual Wester Orchard Pest and Disease Management Conference. 1-8. Annual Western Orchard Pest of Disease Management Conference, 2002 Washington D. C.

INSTITUTO NACIONAL DE SALUD (INS). 2014. Enfermedades transmitidas por vectores. Boletín Epidemiológico. Semana epidemiológica número 20 de 2014 (11 May. al 17 May.). Disponible en: https://www.ins.gov.co/buscador-eventos/BoletinEpidemiologico/2014\%20Boletin\%20epidemiologico\%20semana\%2020.pdf. [Fecha revisión: 19 noviembre 2016].

INSTITUTO NACIONAL DE SALUD (INS). 2016. Enfermedades transmitidas por vectores. Boletín Epidemiológico. Semana epidemiológica número 20 de 2016 (15 may. al 21 may.). Disponible en: https://www.ins.gov.co/buscador-eventos/BoletinEpidemiologico/2016\%20Bolet $\%$ C3\%ADn $\% 20$ epidemiol\%C3\%B3gico\%20semana\%2020.pdf. [Fecha revisión: 19 noviembre 2016].

JARAMILLO-O, N.; DUJARDIN, J. P.; CALLE-LONDOÑO, D. A.; FONSECA-GONZÁLEZ, I. 2015. Geometric morphometrics for the taxonomy of 11 species of Anopheles (Nyssorhynchus) mosquitoes. Medical and Veterinary Entomology 29: 2636. https://doi.org/10.1111/mve.12091

JAMBULINGAM, P.; SADANANDANE, C.; NITHIYANANTHAN, N.; SUBRAMANIAN, S.; ZAIM, M. 2009. Efficacy of novaluron against Culex quinquefasciatus in smalland medium-scale trials, India. Journal of the American Mosquito Control Association 25 (3): 315-322. https://doi.org/10.2987/08-5806.1

KOURI, G. 2006. El dengue, un problema creciente de salud en las Américas. Revista Panamericana Salud Pública 19: 3. https:// doi.org/10.1590/S1020-49892006000300001
LÓPEZ-GATELL, H.; ALPUCHE-ARANDA, C. M.; SANTOSPRECIADO, J.; HERNÁNDEZ-ÁVILA, M. 2016. Dengue vaccine: local decisions, global consequences. Bulletin World Health Organization 16 (94): 850-855. https://doi.org/10.2471/ BLT.15.168765

MAESTRES, R.; REY, V. G.; DE LAS SALAS, A. J.; VERGARA, S. C.; SANTACOLOMA, V. L.; GOENAGA, O. S.; CARRASQUILLA, F. M. 2009. Susceptibilidad de Aedes aegypti (Diptera: Culicidae) a temefos en Atlántico-Colombia. Revista Colombiana de Entomología 35: 202-205.

MINISTERIO DE SALUD Y PROTECCIÓN SOCIAL DE COLOMBIA (MinSalud). 2015. MinSalud confirma primeros nueve casos de zika en Colombia. Disponible en: https://www.minsalud.gov. co/Paginas/Confirmados-primeros-casos-de-virus-del-Zika-enColombia.aspx [Fecha revisión: 27 agosto 2017].

MULLA, M. S.; DARWAZEH, H. A. 1988. Efficacy of new insect growth regulators against mosquito larvae in dairy wastewater lagoons. Journal of the American Mosquito Control Association 4 (3): 322-325.

MULLA, M. S.; THAVARA, U.; TAWATSIN, A.; CHOMPOOSRI, J.; ZAIM, M.; SU, T. 2003. Laboratory and field evaluation of novaluron, a new acylurea insect growth regulator, against Aedes aegypti (Diptera: Culicidae). Journal of Vector Ecology 28 (2): 241-254.

NASCI, R. S. 1986. Relationship between adult mosquito (Diptera: Culicidae) body size and parity in field populations. Environmental Entomology 15 (4): 874-876. https://doi.org/10.1093/ ee/15.4.874

NASCI, R. S. 1987. Adult body size and parity in field populations of the mosquitoes Anopheles crucians, Aedes taeniorhynchus and Aedes sollicitans. Journal of the American Mosquito Control Association 3 (4): 636-637.

NASCI, R. S.; MITCHELL, C. J. 1994. Larval diet, adult size, and susceptibility of Aedes aegypti (Diptera: Culicidae) to infection with Ross River virus. Journal of Medical Entomology 31 (1): 123-126. https://doi.org/10.1093/jmedent/31.1.123

NWANKWO, E. N.; OKONKWO, N. J.; OZUMBA, N. A.; OKAFOR, E. G. 2011. Comparative studies on the larvicidal action of novaluron (Mosquiron ${ }^{\circledR}$ 100EC) and Moringa oliefera (LAM) Seed Oil against Aedes aegypti (Diptera: Culicidae) larvae. African Research Review 5 (1): 424-437. https://doi.org/10.4314/ afrrev.v5i1.64539

OCAMPO, C. B.; SALAZAR-TERREROS, M. J.; MINA, N. J.; MCALLISTER, J.; BROGDON, W. 2011. Insecticide resistance status of Aedes aegypti in 10 localities in Colombia. Acta Tropica 118: 37-44. https://doi.org/10.1016/j.actatropica.2011.01.007

PADILLA, J., ROJAS D.; SÁENZ-GÓMEZ, R. 2012. Dengue en Colombia: epidemiología de la reemergencia a la hiperendemia. Bogotá, Colombia. 281 p.

PANAMERICAN HEALTH ORGANIZATION (PAHO). 2008. Reports cases of dengue and dengue hemorrhagic fever (DHF), region of the Americas (by country and subregion). Washington, DC, Pan American Health Organization 78 (3): 364-369.

RAWLINS, S. C.; WAN, J. O. 1995. Resistance in some Caribbean populations of Aedes aegypti to several insecticides Journal of the American Mosquito Control Association 11: 59-65.

RINCÓN SILVA, N. G.; RINCÓN SILVA, J. D. 2016. Impacto general de las fiebres del Zika y Chikungunya en Colombia y América del Sur: Análisis general de los virus y su importancia. Biociencias. Revista de la Facultad de Ciencias de la Salud de la Universidad Libre Seccional Barranquilla 11 (2): 77-93. https:// doi.org/10.18041/2390-0512/bioc..2.2566

RUIZ-LÓPEZ, F.; GONZÁLEZ-MAZO, A.; VELEZ-MIRA, A.; GÓMEZ, G.; ZULETA, L. 2016. Presencia de Aedes (Stegomyia) aegypti (Linneaus, 1762) y su infección natural con el virus del dengue en alturas no registradas para Colombia. Biomédica 36 (2): 303-308. http://dx.doi.org/10.7705/biomedica.v36i2.3301

SALAZAR, M.; CARVAJAL, A.; CUELLAR, M.; OLAYA, A.; QUIÑONES, J. 2007. Resistencia a insecticidas en poblaciones 
de Aedes aegypti y Anopheles spp. en los departamentos de Huila, Valle, Cauca y Nariño. Memorias XIII Congreso colombiano de parasitología y medicina tropical. Biomédica 27 (2): 177.

SAN MARTIN, J. L. 2009. Dengue, guías para el diagnóstico, tratamiento prevención y control. [Monografía en internet]. Organización Mundial de la Salud (OMS) Programa especial para la investigación y capacitación de enfermedades tropicales (TDR). Disponible en: http://www2.paho.org/HQ/dmdocuments/2011/ ndeng31570.pdf. [Fecha revisión: 28 enero 2015].

SANTACOLOMA, L.; BROCHERO, H.; CHÁVEZ, B. 2007. Estado de la susceptibilidad a insecticidas de Aedes aegypti en cinco departamentos de Colombia. Memorias XIII Congreso colombiano de parasitología y medicina tropical. Biomédica 27 (2): 175.

SANTACOLOMA, L.; CHAVES, B.; BROCHERO, H. 2012. Estado de la susceptibilidad de poblaciones naturales del vector del dengue a insecticidas en trece localidades de Colombia. Biomédica 32 (3): 333-343. http://dx.doi.org/10.7705/biomedica.v32i3.680

SISTEMA DE INFORMACIÓN PARA LA VIGILANCIA EN SALUD PÚBLICA (SIVIGILA). 2014. Datos Sistema de Vigilancia en Salud Pública SIVIGILA 2014. Instituto Nacional de Salud, Ministerio de Salud y Protección Social, Bogotá, D. C., Colombia. Disponible en: https://www.datos.gov.co/Salud-yProtecci-n-Social/SIVIGILA-2014/b22h-2un2 [Fecha revisión: 30 agosto 2017].

SUÁREZ, M. F.; GONZÁLEZ, R.; MORALES, C. 1996. Temefos resistance to Aedes aegypti in Cali, Colombia. $45^{\text {a }}$ Annual meeting of the American Society of Tropical Medicine and Hygiene, 110 Baltimore, Maryland. Supplement to the American Journal of Tropical Medicine and Hygiene 55: 257.

SUMANOCHITRAPON, W.; STRICKMAN, D.; SITHIPRASASNA, R.; KITTAYAPONG, P.; INNIS, B. 1998. Effect of size and geographic origin of Aedes aegypti on oral infection with dengue-2 virus. American Journal of Tropical Medicine Hygiene 58 (3): 283-286. https://doi.org/10.4269/ajtmh.1998.58.283
TAWATSIN, A.; THAVARA, U.; BHAKDEENUAN, P.; CHOMPOOSRI, J.; SIRIYASATIEN, P.; ASAVADACHANUKORN, P.; MULLA, M. S. 2007. Field evaluation of novaluron, a chitin synthesis inhibitor larvicide, against mosquito larvae in polluted water in urban areas of Bangkok, Thailand. Southeast Asian Journal of Tropical Medicine and Public Health 38 (3): 434-441.

WORLD HEALTH ORGANIZATION (WHO). 2005a. Guidelines for laboratory and field testing of mosquito larvicides. WHO/ CDS/WHOPES/GCDPP/2005.13. WHO, Communicable Disease Control, Prevention and Eradication WHO Pesticide Evaluation Scheme $41 \mathrm{p}$.

WORLD HEALTH ORGANIZATION (WHO). 2005b. Report of the eighth WHOPES working group meeting. Review of novaluron $10 \%$ EC. WHO/CDS/WHOPES/2005.10. Ginebra. WHO 42 p.

\section{Origen y financiación}

Este estudio hizo parte del proyecto de investigación para la evaluación de insecticidas reguladores de crecimiento y fue financiado por Adama Andina B.V. Sucursal Colombia.

\section{Contribución de los autores}

Marcela Quimbayo F.: Desarrollo de la idea, colecta, tabulación e interpretación de los datos y escritura del manuscrito.

Juliana Pérez-Pérez: Interpretación de datos, análisis estadistico y escritura del manuscrito.

Paola A. Rodríguez-Gaviria: Desarrollo de la idea, análisis de la información y revisión del manuscrito.

Juan David Amaya: Desarrollo de la idea, análisis de la información y revisión del manuscrito.

Guillermo L. Rúa-Uribe: Concibió la idea, seguimiento al proceso de investigación, análisis de los resultados, escritura y revisión del manuscrito. 